\title{
Cortical network underlying speech production during delayed auditory feedback
}

\author{
Muge Ozker ${ }^{1 *}$, Werner Doyle ${ }^{2}$, Orrin Devinsky ${ }^{1}$, Adeen Flinker ${ }^{1}$
}

1. Department of Neurology, New York University Langone Medical Center, NY, USA

2. Department of Neurosurgery, New York University Langone Medical Center, NY, USA

* Corresponding Author: Muge Ozker

Email: muge.sertel@nyulangone.org 


\begin{abstract}
Hearing one's own voice is critical for fluent speech production as it allows for the detection and correction of vocalization errors in real-time. This behavior known as the auditory feedback control of speech is impaired in various neurological disorders ranging from stuttering to aphasia, however the underlying neural mechanisms are still poorly understood. Computational models of speech motor control suggest that, during speech production, the brain uses an efference copy of the motor command to generate an internal estimate of the speech output. When actual feedback differs from this internal estimate, an error signal is generated to correct the estimate and subsequent motor commands to produce intended speech. We were able to localize these neural markers using electrocorticographic recordings from neurosurgical subjects during a delayed auditory feedback (DAF) paradigm. In this task, subjects hear their voice with a time delay as they produced words and sentences (similar to an echo on a conference call), which is well known to disrupt fluency by causing slow and stutter-like speech in humans. We observed a significant response enhancement in auditory cortex that scaled with the duration of feedback delay indicating an auditory speech error signal. Immediately following auditory cortex, dorsal precentral gyrus (dPreCG), a region that has not been implicated in auditory feedback processing before, exhibited a markedly similar response enhancement suggesting a tight coupling between the two regions. Critically, response enhancement in $\mathrm{dPreCG}$ occurred only when subjects profoundly slowed down their speech during articulation of long utterances due to a continuous mismatch between produced speech and reafferent feedback. These results suggest that dPreCG plays an essential role in updating the internal speech estimates to maintain fluency as well as coordinating the efference copy and auditory error signals during speech production.
\end{abstract}




\begin{abstract}
Abbreviations: DAF = Delayed auditory feedback; $\mathrm{dPreCG}=$ Dorsal precentral gyrus; DTW $=$ Dynamic time warping $; \mathrm{ECoG}=$ Electrocorticography $\mathrm{IFG}=$ Inferior frontal gyrus; $\mathrm{NMF}=$ Non-negative matrix factorization; PostCG = Postcentral gyrus; $\mathrm{SMG}=$ Supramarginal gyrus; $\mathrm{STG}=$ Superior temporal gyrus; $\mathrm{vPreCG}=$ Ventral precentral gyrus;
\end{abstract}

\title{
Introduction
}

Human speech production is strongly influenced by the auditory feedback it generates. When we speak, we continuously monitor our vocal output and adjust our vocalization to maintain fluency. For example, speakers involuntarily raise their voice to be more audible when auditory feedback is masked in the presence of background noise [1, 2]. Similarly, when speakers hear themselves with a delay (e.g. voice delays or echoes in teleconferencing), they compensate for the auditory feedback delay by slowing down and resetting their speech. This compensatory adjustment of human vocalization provides evidence for a mechanism which detects and corrects vocal errors in real-time. Abnormal auditory feedback control has been implicated in various disorders including stuttering, aphasia, Parkinson's disease, autism spectrum disorder and schizophrenia [3-7], however the neural underpinnings of this dynamic system remain poorly understood.

Predictive models of speech motor control suggest that the brain generates an internal estimate of the speech output during speech production using an efference copy of the vocalmotor command. When there is a mismatch between this internal estimate and the perceived (reafferent) auditory feedback, the auditory response is enhanced to encode the mismatch. This auditory error signal is then relayed to vocal-motor regions for the real-time correction of vocalization in order to produce the intended speech [8-10]. 
In support of these models, electrophysiological studies in non-human primates demonstrated increased activity in auditory neurons when the frequency of the auditory feedback is shifted during vocalization [11]. Behavioral evidence in human studies showed that when formant frequencies of a vowel or the fundamental frequency (pitch) is shifted, speakers change their vocal output in the opposite direction of the shift to compensate for the spectral perturbation [12-14]. In line with non-human primate studies, human neurosurgical recordings as well as neuroimaging studies demonstrated that these feedback-induced vocal adjustments are accompanied by enhanced neural responses in auditory regions [15-17].

An alternative method to manipulating the spectral features of auditory feedback is altering its temporal features by delaying the voice onset in real time, termed "delayed auditory feedback (DAF)". First described in the 1950s, DAF strongly disrupts speech fluency leading to slower speech rate, pauses, syllable repetitions and increased voice pitch or intensity [18-20]. Further, higher susceptibility to DAF occurs in autism spectrum disorder, non-fluent primary progressive aphasia, schizophrenia and other neurological disorders [4-6]. Interestingly, DAF improves speech fluency in individuals who stutter and is a therapeutic approach in speech therapy for stuttering and Parkinson's Disease [21-23]. Although these behavioral effects have been widely studied in both normal and clinical groups, only a few neuroimaging studies have investigated the neural responses. Studies have demonstrated enhanced responses in bilateral posterior superior cortices during delayed feedback compared with normal auditory feedback conditions [24-26]. However, the exact temporal dynamics and spatial distribution of the cortical networks underlying speech production and reafferent feedback processing remain unknown.

To address this issue, we leveraged the excellent spatial and temporal resolution of electrocorticography (ECoG). Using ECoG, we acquired direct cortical recordings from 15 
epilepsy patients while they read aloud words and sentences. As they spoke, we recorded their voice and played it back to them through earphones either simultaneously or with a delay (50, 100 and 200 milliseconds). Behaviorally, we found that subjects slowed down their speech to compensate for the delay, and did so more profoundly when producing sentences. Neurally, there was a significant increase in activity across a large speech network encompassing temporal, parietal and frontal sites that scaled with the duration of feedback delay. Critically, when speech was slowed down and became effortful, the dorsal division of the precentral gyrus was preferentially recruited at an early timing to support ongoing articulation. To our knowledge, we introduce the first temporally delayed feedback processing investigation with invasive human electrophysiology in which we reveal the fine-grained spatiotemporal dynamics of the neural mechanisms underlying compensatory adjustment of human vocalization.

\section{Results}

Subjects $(\mathrm{N}=15)$ performed a word-reading task (single 3-syllable words) while their voice onset was delayed (no delay, 50, 100 and $200 \mathrm{~ms}$ ) and played back to them through earphones in real time, a paradigm known as delayed auditory feedback (DAF). We first analyzed the voice recordings of subjects and measured the articulation duration at different amount of delays to establish the behavioral effect of DAF (Fig 1A). Articulation duration increased slightly with delay: average articulation duration across subjects was $0.698,0.726$, 0.737 and 0.749 milliseconds for no delay, 50, 100 and 200 milliseconds delay conditions respectively. While this increase was not significant (Fig 1B, ANOVA: $F=1.985 \mathrm{p}=0.165)$ we observed robust neural changes. 
A

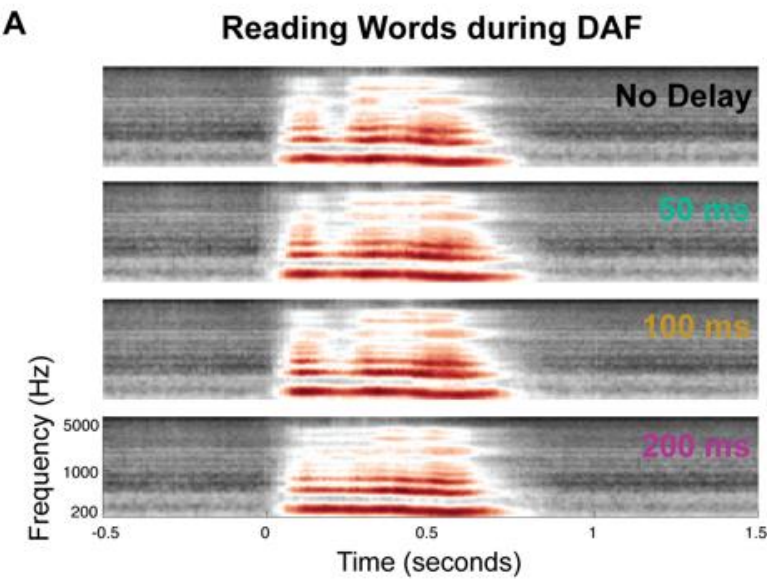

C

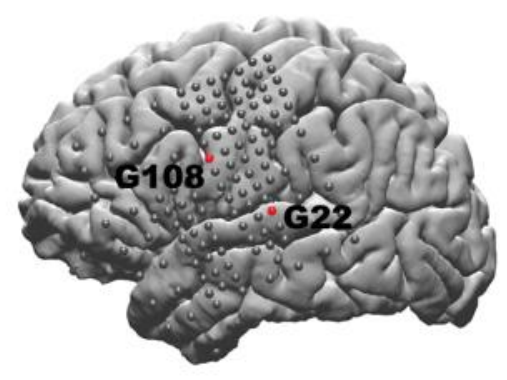

D
B
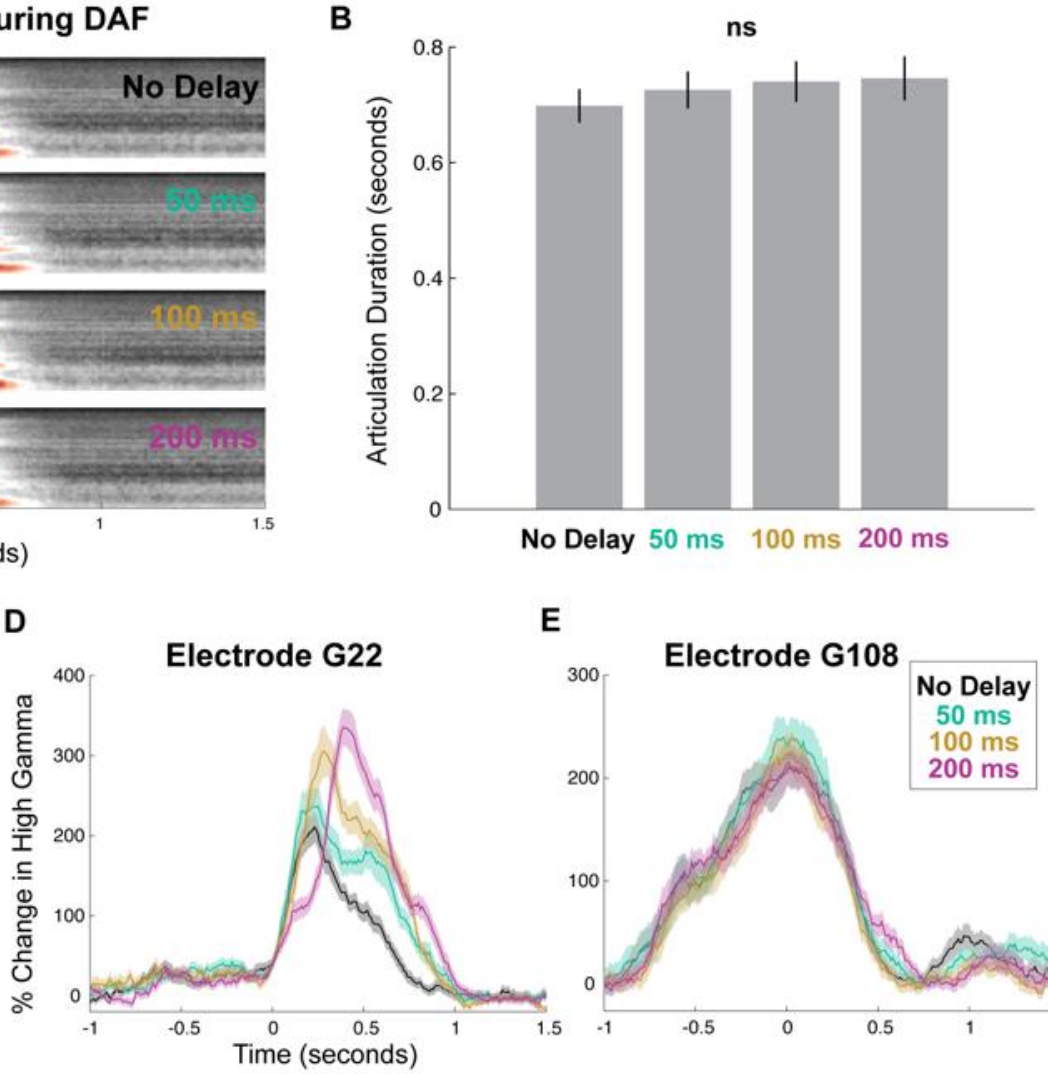

E

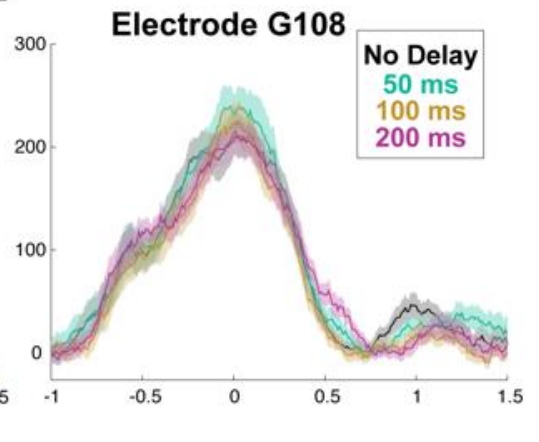

Fig 1. Behavioral and neural responses during word-reading with DAF.

(A) Speech spectrogram of a single subject articulating words during DAF conditions. (B) Mean articulation duration of words during DAF conditions averaged across subjects. Error bars show SEM over subjects. (C) Cortical surface model of the left hemisphere brain of a single subject. Gray circles indicate the implanted electrodes. Red highlighted electrodes are located on the STG (G22) and on the vPreCG (G108). (D) High gamma responses in an auditory electrode (G22) to articulation of words during DAF conditions (color coded). Shaded regions indicate SEM over trials. (E) High gamma responses in a motor electrode (G108) to articulation of words during DAF conditions (color coded). Shaded regions indicate SEM over trials. SEM = Standard error of the mean

To quantify the neural response, we used the high gamma broadband signal $(70-150 \mathrm{~Hz}$, see Methods), a widely used index of cortical activity which correlates with underlying neuronal spike rates [30-32]. Two response patterns emerged among the electrodes that showed significant activity during speech production (see Electrode Selection in Methods). In the first pattern, shown on a representative auditory electrode located in the STG (Fig 1C), neural response started after speech onset and its amplitude increased significantly with delay (Fig 1D, ANOVA: 
$\left.\mathrm{F}=37, \mathrm{p}=1.55 \times 10^{-8}\right)$. In the second pattern, shown on a representative motor electrode located in vPreCG (Fig 1C), neural response started before speech onset and its amplitude was not affected by delay (Fig 1E, ANOVA: $F=0.084, p=0.772$ ). This result demonstrated that although DAF did not significantly affect speech behavior (i.e. articulation duration), it affected the neural response in auditory sites that are involved in speech processing.

To characterize the two major response patterns in the brain we chose to use an unbiased, data-driven approach which does not impose any assumptions or restrictions on the selection of responses. We performed an unsupervised clustering analysis using the NMF algorithm on neural responses across all delay conditions, brain sites and subjects [29, 33]. The clustering analysis identified the major response patterns represented by two distinct clusters, which corroborated our representative results shown in a single subject (Fig 1C-E) as well as visual inspection of the data across subjects. The first response pattern (Cluster $1, \mathrm{~N}=125$ electrodes) started after speech onset and peaked at $320 \mathrm{~ms}$ reaching 115 percent change in amplitude. The second response pattern (Cluster 2, $\mathrm{N}=253$ electrodes) started much earlier approximately 750 $\mathrm{ms}$ prior to speech onset and peaked $140 \mathrm{~ms}$ after speech onset reaching 60 percent change in amplitude (Fig 2A). These two clusters had a distinct anatomical distribution (Fig 2B): Cluster 1 was mainly localized to STG suggesting an auditory function while Cluster 2 was localized to frontal cortices suggesting a pre-motor and motor function. 
A

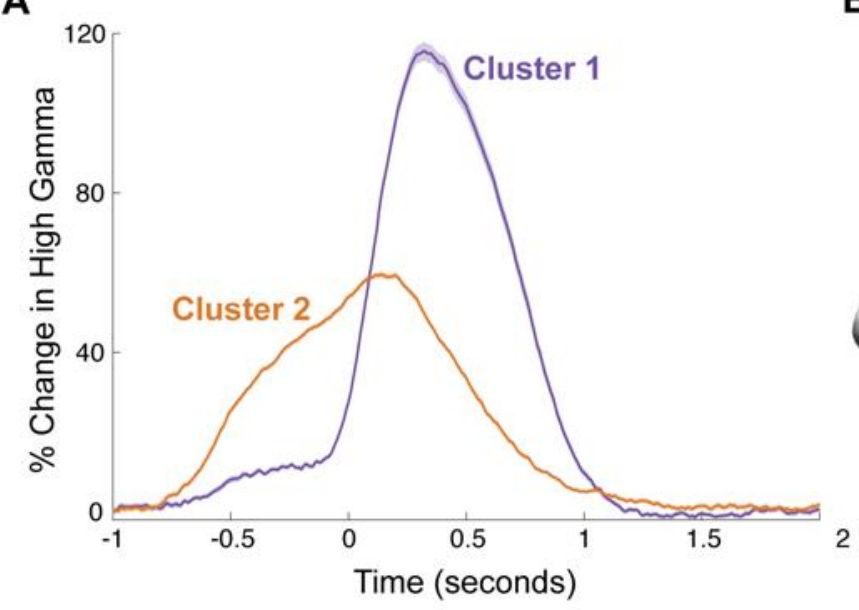

C

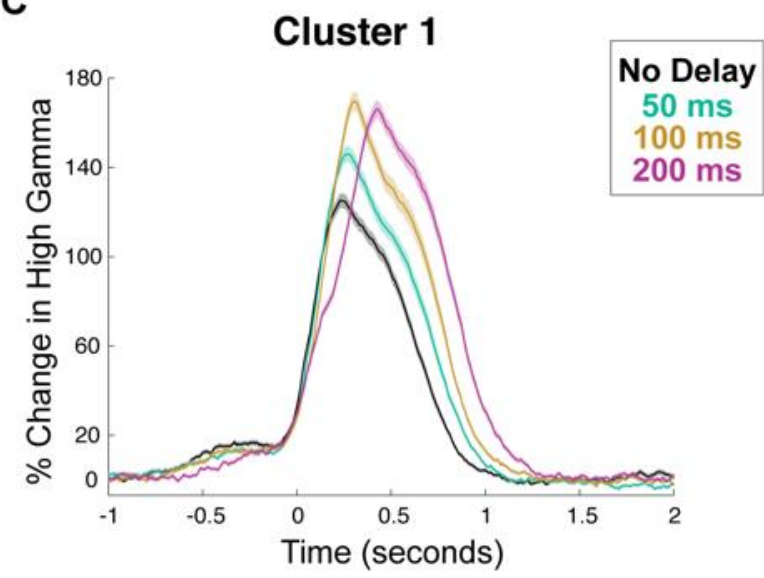

B

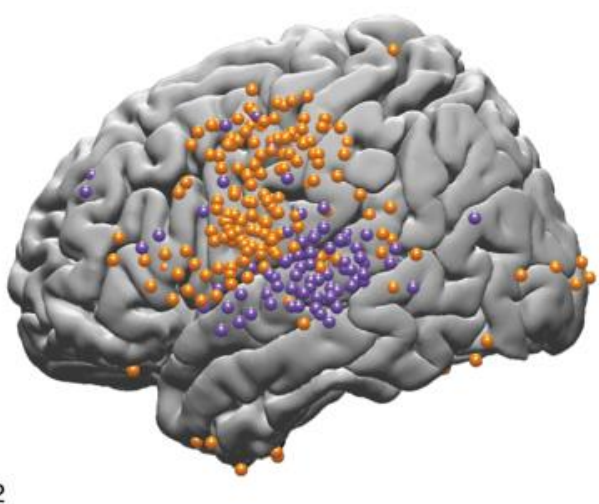

D

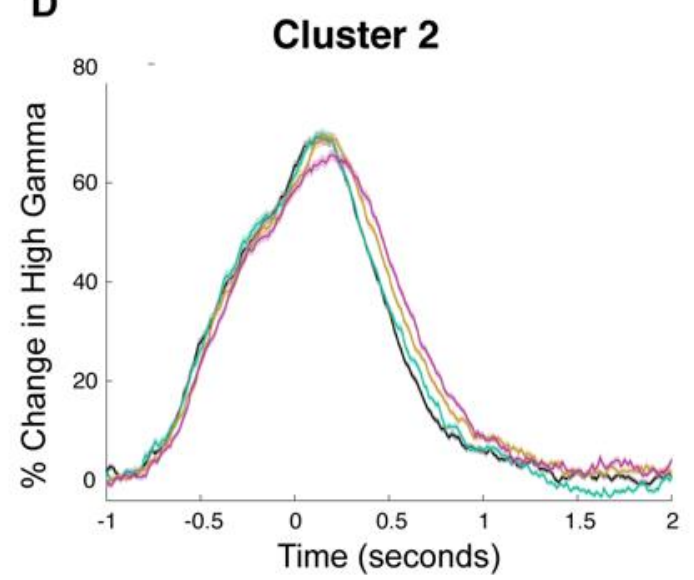

Fig 2. Clustering with non-negative matrix factorization.

(A) High gamma responses averaged across electrodes in the two clusters provided by the unsupervised NMF. Shaded regions indicate SEM over trials. (B) Spatial distribution on cortex of electrodes in the two clusters displayed on the left hemisphere of a template brain. (C) High gamma responses to articulation of words during DAF conditions averaged across electrodes in Cluster 1. Shaded regions indicate SEM over trials. (D) High gamma response to articulation of words during DAF averaged across electrodes in Cluster 2. Shaded regions indicate SEM over trials. SEM = Standard error of the mean

Next, we examined the effect of DAF on these two clusters. The amplitude of the neural response increased significantly with delay in Cluster 1 (Fig 2C, ANOVA: F = 5.35, p = 0.02), but not in Cluster 2 (Fig 2D, ANOVA: $\mathrm{F}=1.65, \mathrm{p}=0.2$ ). The duration of the neural response did not show a significant increase in either of the clusters (ANOVA: $F=1, p=0.32$ for Cluster 1 and $\mathrm{F}=0.01, \mathrm{p}=0.92$ for Cluster 2 ). 
Reading words with DAF did not prolong articulation duration and while it increased neural responses in auditory regions, it did not affect responses in motor regions. We hypothesized that a longer and more complex stimulus may elicit a stronger behavioral response and motor regions will show an effect of DAF when articulation is strongly affected. To test this prediction, we performed another experiment in which subjects read aloud sentences during DAF. Indeed, articulating longer speech segments (8-word sentences) during DAF resulted in a significantly stronger behavioral effect (Fig 3A). Articulation duration increased significantly with delay: average articulation duration across subjects was 2.761, 2.942, 3.214 and 3.418 seconds for no delay, 50, 100 and 200 milliseconds delay conditions respectively (Fig 3B, ANOVA: $F=17.11, p=0.0001)$. 
A

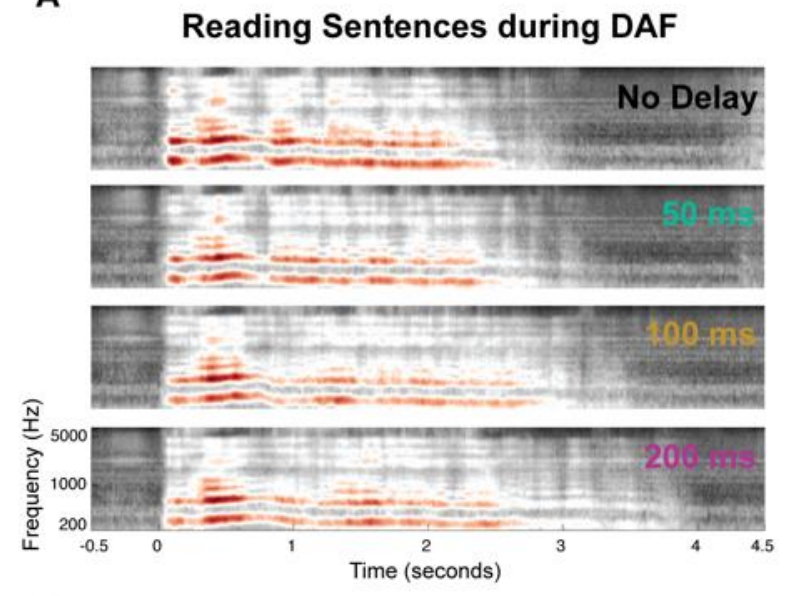

B

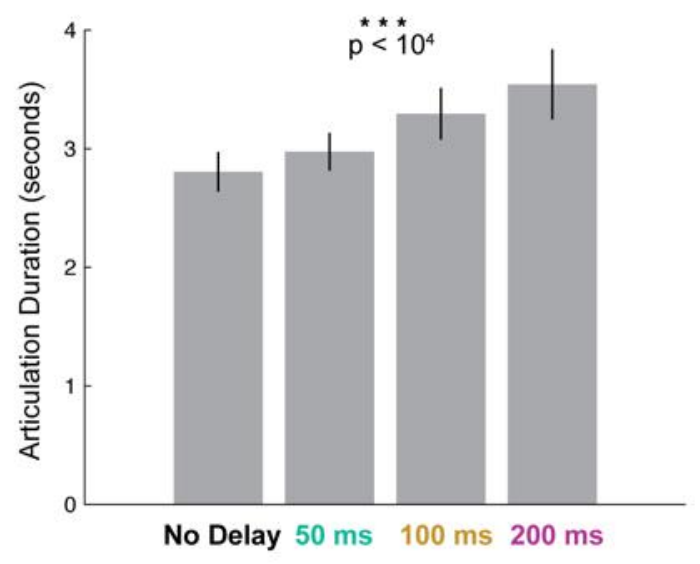

D
C

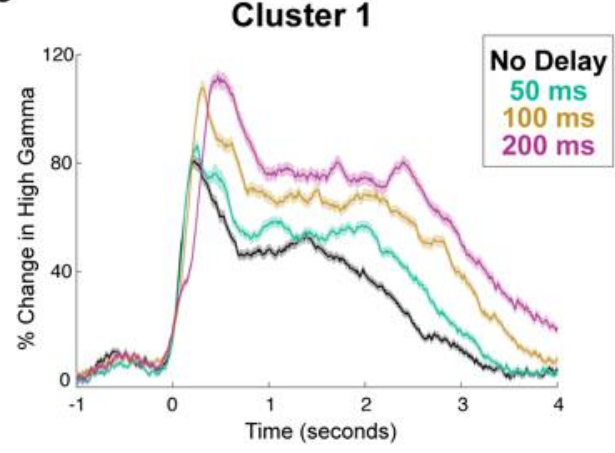

Cluster 2

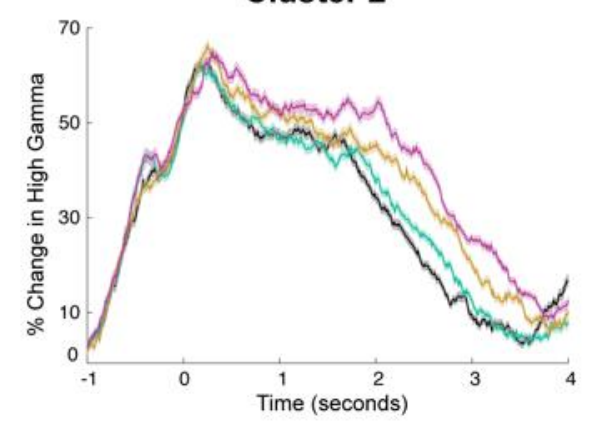

E

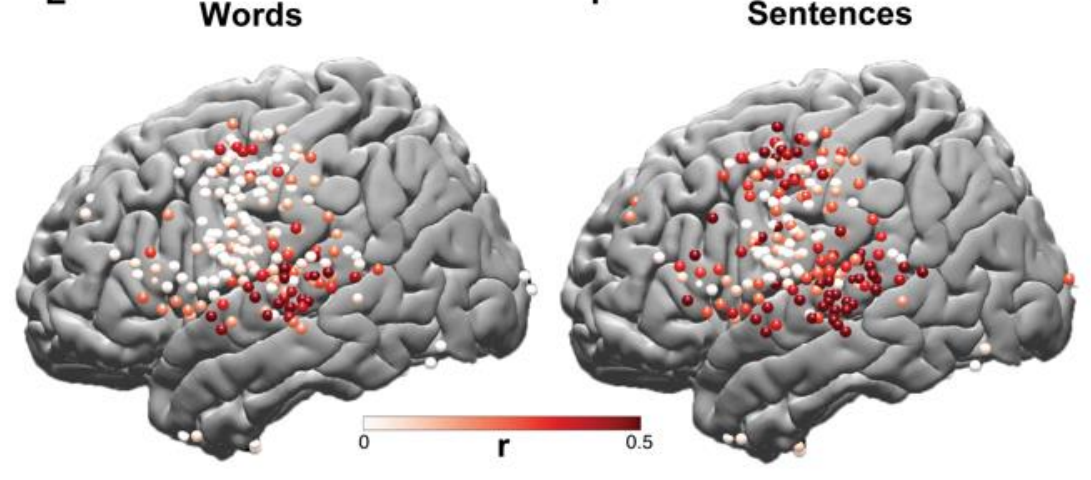

G

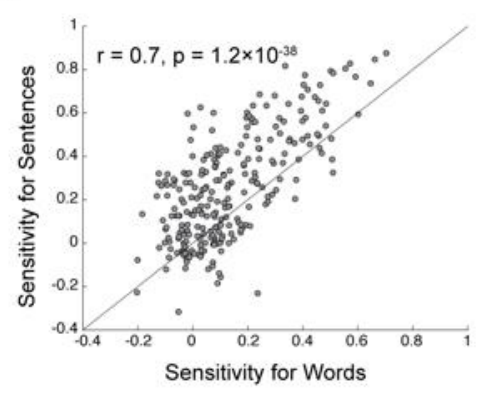

Fig 3. Behavioral and neural responses during sentence-reading with DAF.

(A) Speech spectrogram of a single subject articulating sentences during DAF conditions showing a marked increase in articulation duration. (B) Mean articulation duration of sentences during DAF conditions averaged across subjects showing a significant effect of duration. Error bars show SEM over subjects. (C) High gamma responses to articulation of sentences during DAF conditions averaged across electrodes in Cluster 1. Shaded regions indicate SEM over trials. (D) High gamma responses to articulation of sentences during DAF conditions averaged across electrodes in Cluster 2. Shaded regions indicate SEM over trials. (E) Anatomical map of electrodes across all subjects displayed on the left hemisphere of a template brain showing the neural sensitivity to DAF during word-reading. (F) Anatomical map of electrodes across all subjects displayed on the left hemisphere of a template brain showing the neural sensitivity to DAF during sentences-reading. (G) Scatter plot and fitted regression showing significant correlation between sensitivity to DAF for the word-reading and sentence-reading tasks. Each circle represents one electrode. SEM = Standard error of the mean 
Next, we examined the neural response to DAF in the two electrode clusters we identified previously (Fig 2B). When reading words during DAF, amplitude of the neural response increased with delay in Cluster 1 but not in Cluster 2 (Fig 2C and 2D). However, when reading sentences during DAF, neural response in both clusters showed a sustained effect (Fig 3C and 3D, ANOVA: $\mathrm{F}=18, \mathrm{p}=2.95 \times 10^{-5}$ for Cluster 1 and $\mathrm{F}=4.8, \mathrm{p}=0.03$ for Cluster 2$)$. Also, when reading words during DAF, duration of the neural response in neither of the clusters showed a significant effect of delay. However, when reading sentences during DAF, neural response duration in both clusters increased significantly with delay paralleling the significant behavioral effect of DAF on articulation duration (ANOVA: $F=21.6, p=10^{-5}$ for Cluster 1 and $\mathrm{F}=35.5, \mathrm{p}=10^{-8}$ for Cluster 2$)$

Our clustering analysis identified two response components that were mostly anatomically distinct reflecting an auditory response to self-generated speech and a motor response to articulation. The auditory component was unique in exhibiting an enhanced response during both word-reading and sentence-reading with DAF likely representing an auditory error signal. Response enhancement changed as a function of feedback delay, which suggests that auditory error signal does not simply encode the mismatch between intended and perceived speech but is sensitive to the amount of mismatch. We quantified the error signal by calculating a sensitivity index for each electrode by measuring the trial-by-trial correlation between the delay and the neural response averaged over a 0 to $1 \mathrm{~s}$ time window for words and over 0 to $3 \mathrm{~s}$ for sentences. A large sensitivity value indicated a strong response enhancement with increasing delays.

Comparing sensitivity indices for word-reading and sentence-reading tasks revealed that electrodes that were sensitive to DAF for the word-reading task were also sensitive for the 
sentence-reading task $\left(r=0.7, p=1.2 \times 10^{-38}\right.$, Fig 3G), however the majority of electrodes showed larger sensitivity to DAF for the sentence-reading task. Moreover, several sites such as IFG and dPreCD showed increased sensitivity in the sentence-reading task (Fig 3E-F). This result suggests that articulating longer speech segments during DAF results in stronger overall sensitivity across auditory and motor regions and engages a larger brain network uniquely recruiting additional frontal regions.

We further examined the neural response to DAF in six different regions of interest based on within subject anatomy: STG, vPreCG, dPreCG, postCG, SMG and IFG (Fig 4A-F). Comparing sensitivity indices for word-reading and sentence-reading tasks in these regions revealed that all six regions showed larger sensitivity to DAF during sentence-reading (unpaired ttest; STG: $\mathrm{t}=3.13, \mathrm{p}=0.0025, \mathrm{vPreCG}: \mathrm{t}=4.55, \mathrm{p}=2.7 \times 10^{-5}, \mathrm{dPreCG}: \mathrm{t}=5.15, \mathrm{p}=3 \times 10^{-6}$, postCG: $\mathrm{t}=3.1, \mathrm{p}=0.0024, \mathrm{SMG}: \mathrm{t}=1.87, \mathrm{p}=0.07$, IFG: $\mathrm{t}=2.89, \mathrm{p}=0.0069 ;$ Fig 4G). To reveal how response enhancement to DAF changed across time during the sentence-reading task, we performed a one-way ANOVA at each time point (see Methods) and marked the timepoints when the neural response to the four delay conditions were significantly different for at least 200 consecutive milliseconds. Significant divergence onset during sentence-reading started the earliest in STG at $80 \mathrm{~ms}$ after speech onset, followed by dPreCG at $350 \mathrm{~ms}$ and SMG gyrus at $680 \mathrm{~ms}$, and lasted throughout the stimulus. In postCG, vPreCG and IFG, responses diverged much later, at 1.82, 1.87 and 2.30 s respectively (Fig $\mathbf{4 H}$ ). These timings reflect when cortical regions become sensitive to DAF and provide evidence for two distinct timeframes of early (STG, dPreCG, SMG) and late (postCG, vPreCG, IFG) recruitment. 

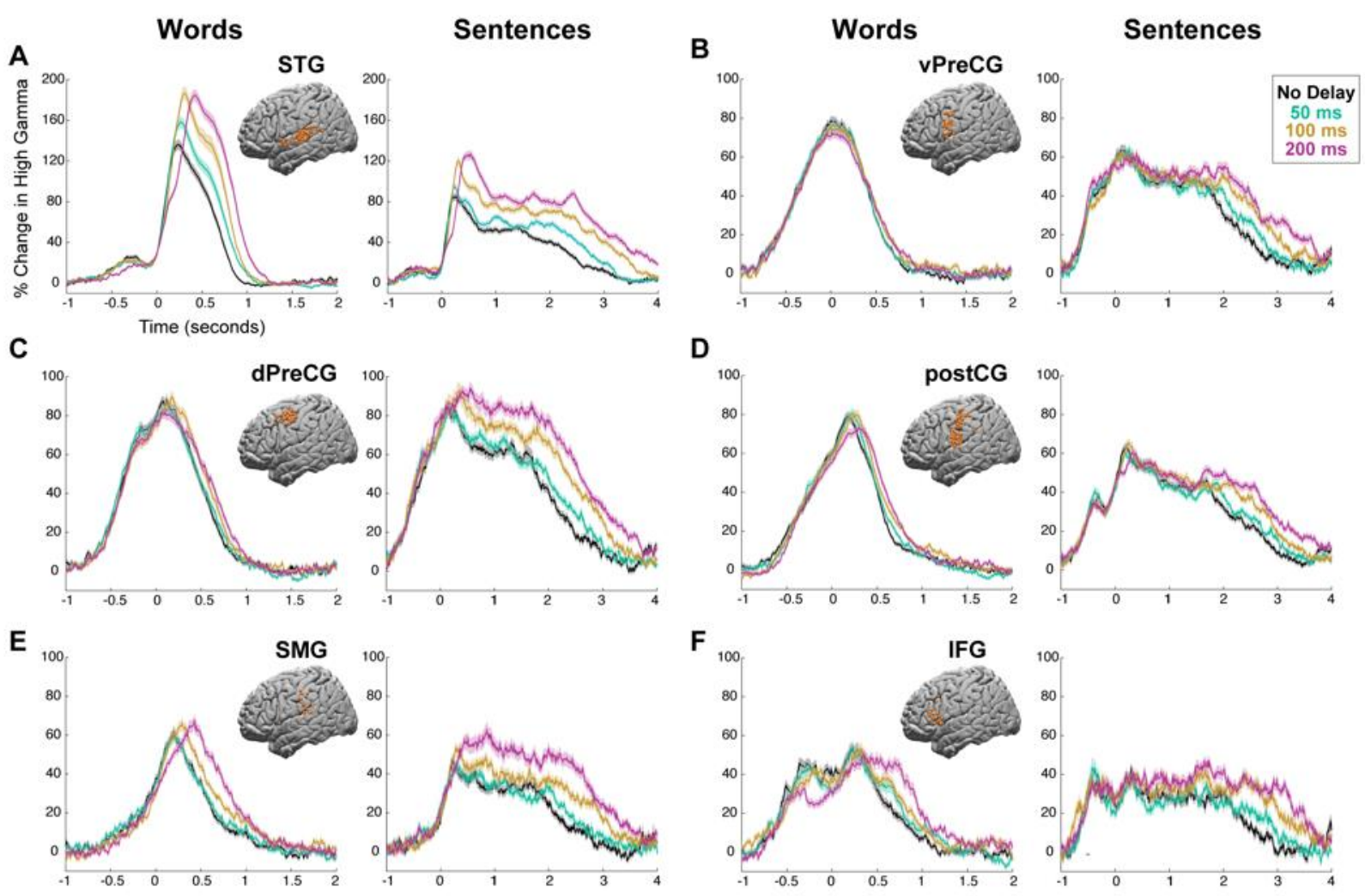

G

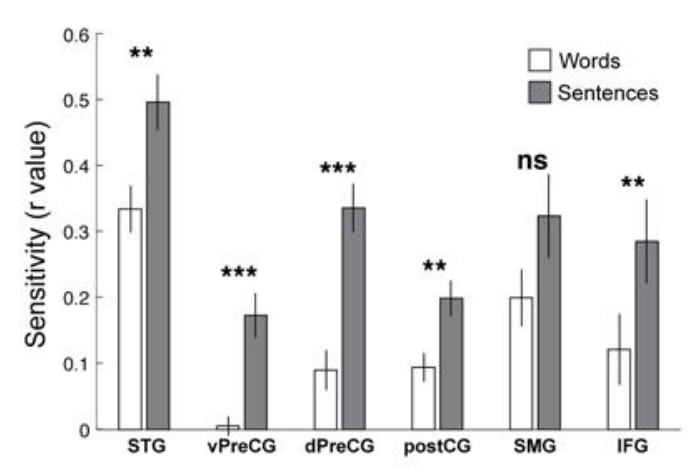

H

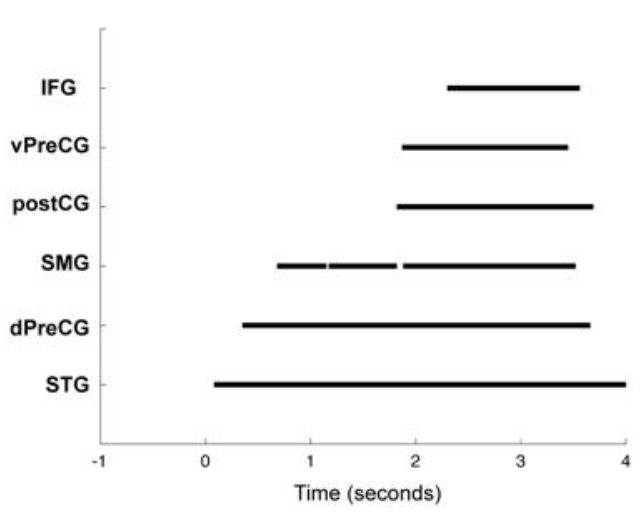

Fig 4. Neural responses to DAF by regions.

(A-F) High gamma responses to articulation of words and sentences during DAF in six different regions: STG (a), vPreCG (b), dPreCG (c), postCG (d), SMG (e) and IFG (f). Inset brain figures shows the location of electrodes across all subjects on the left hemisphere of a template brain. Colors represent the various DAF conditions and shaded regions indicate SEM over trials. (G) Sensitivity to DAF during word-reading and sentence-reading tasks averaged across electrodes (error bars indicate SEM over electrodes) in six different regions. (H) Time intervals when the neural response to reading sentences during DAF diverged significantly across conditions within each of the six different regions. Significance is assessed using a shuffled permutation approach. SEM = Standard error of the mean 
Examining different regions of the speech network revealed variable degree of neural response enhancement to DAF. The increase in response amplitude was usually accompanied by an increase in response duration, which was a result of longer articulation duration. In order to disentangle the enhanced neural response representing an error signal with longer articulation duration due to the exerted behavior, we applied a temporal normalization technique. We transformed the neural response time series using dynamic time warping (DTW) so that they would match in time span. DTW measures the similarity between two temporal sequences with different lengths by estimating a distance metric (a warping path) that would transform and align them in time. Matching the neural responses in time allowed us to directly compare their amplitudes and identify which brain regions produce an error signal in response to DAF rather than just sustained activity in time due to longer articulation.

We compared two conditions, which show the largest neural response difference in terms of amplitude and duration; 0 and 200 ms delay conditions (see Dynamic Time Warping Analysis in Methods). After aligning the responses in time, we averaged the amplitudes of the timewarped signals over time (0-6 s) and compared the two conditions by running an unpaired t-test. Amplitudes of the time-warped responses to DAF were significantly larger in $\mathrm{STG}(\mathrm{t}=5.5, \mathrm{p}=$ $\left.7.7 \times 10^{-8}\right), \operatorname{SMG}(\mathrm{t}=3.06, \mathrm{p}=0.0025), \mathrm{dPreCG}(\mathrm{t}=2.4, \mathrm{p}=0.016)$ and IFG $\left(\mathrm{t}=3.4, \mathrm{p}=10^{-3}\right)$ but not in ${ }_{\text {PreCG }}(\mathrm{t}=0.82, \mathrm{p}=0.41)$ and postCG $(\mathrm{t}=1.66, \mathrm{p}=0.0971)$ regions $($ Fig 5A-F $)$. Lastly, to examine the spatial distribution of the (time-warped) error response in more detail, we calculated the percent increase in response amplitude in single electrodes (HGB200 - HGB no delay $/ ~$ $\mathrm{HGB}_{\text {no delay }} * 100$ ). This analysis revealed that the magnitude of the error response was variable both across and within the regions of the speech network. Overall the error signal centered around four major cortical networks: STG, IFG, SMG and dPreCG (Fig 5G). 

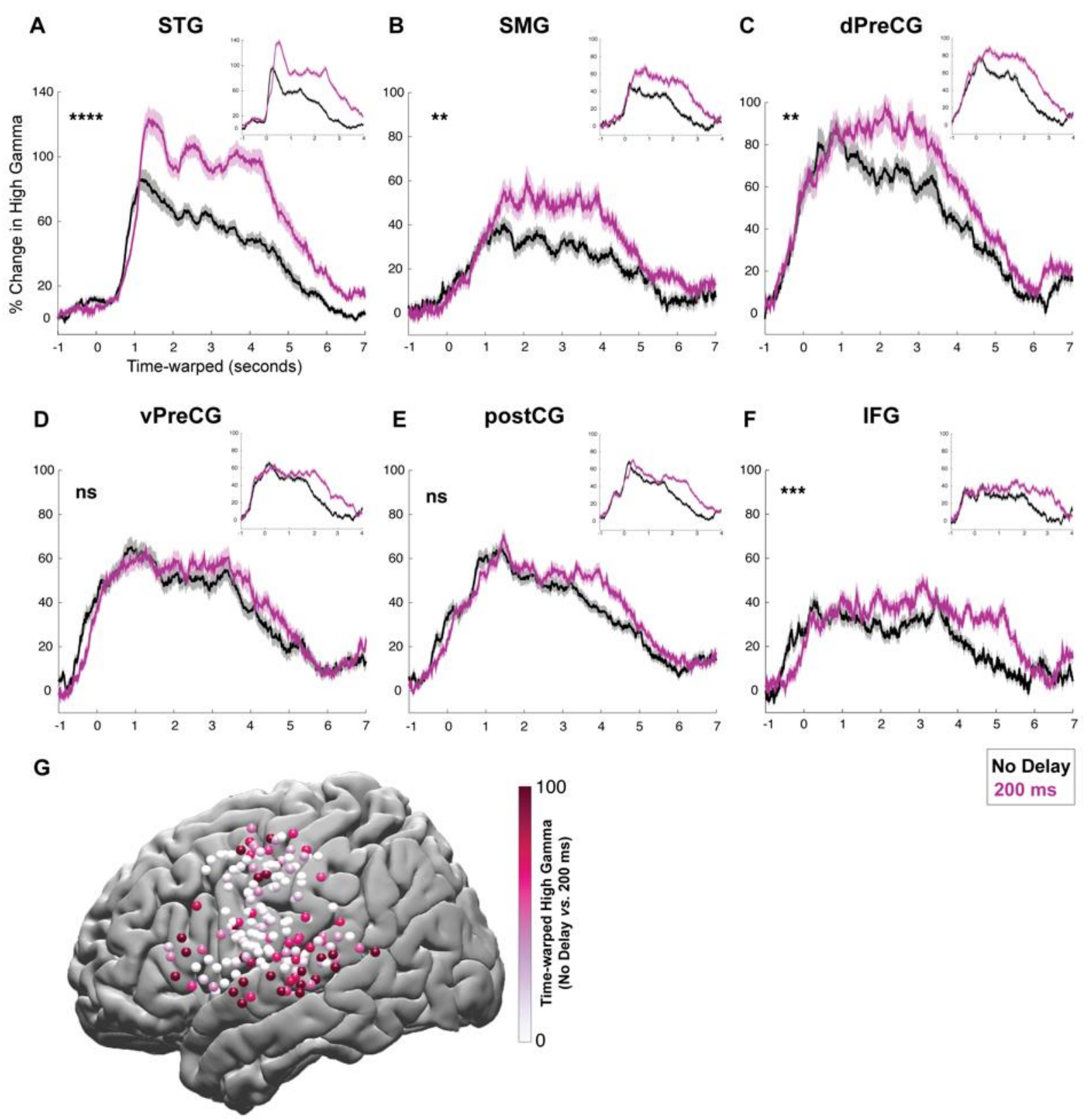

No Delay $200 \mathrm{~ms}$

Fig 5. Time warped neural responses during sentence-reading with DAF.

(A-F) High gamma responses after correction for articulation duration using dynamic time warping. Activity locked to articulation of sentences is shown for no delay (black) and $200 \mathrm{~ms}$ delay (magenta) conditions in six different regions: STG, SMG, vPreCG, dPreCG, postCG and IFG. Inset figures show the uncorrected high gamma responses which include the normal articulation timing. (G) Spatial distribution of the increase in neural responses to sentencereading during DAF (200 ms) compared to no delay condition. Electrodes across all subjects are displayed on the left hemisphere of a template brain. 


\section{Discussion}

Artificially slowing down speech when hearing one's own delayed voice provides a strong framework to investigate how auditory feedback influences the motor control of speech. Our study is one of the few electrophysiological investigations [34,35], and to our knowledge, the only ECoG investigation of the underlying neural substrates. We compared the effects of DAF on producing short versus long speech segments by using word and sentence stimuli and showed that producing sentences during DAF had a stronger disruptive effect on speech. We used an unsupervised clustering algorithm (NMF) to determine auditory and motor regions involved in speech production and then identified four subregions of the speech network that are centrally engaged in the processing of auditory feedback: STG, SMG, dPreCG and IFG. The exquisite resolution of ECoG provided us with the precise spatiotemporal evolution of feedback processing in these distinct regions. Neural responses were enhanced in amplitude and extended in duration for large delays reflecting the error signal caused by altered feedback and the subsequent longer articulation. To dissociate the error signal from the effect of prolonged articulation, we used dynamic time warping algorithm and temporally aligned the neural signals with the patients' speech acoustics. We found that dPreCG showed response enhancement immediately after auditory cortex when speech fluency was strongly disrupted during production of sentences with DAF. These results highlighted $\mathrm{dPreCG}$ as a critical region for maintaining speech fluency when dynamic auditory feedback processing is required to produce longer utterances.

During speech production, the reafferent (perceived) auditory feedback is not immediately useful due to noise and delays in neural processing (axonal transmission, synaptic processes etc.). According to predictive models of speech motor control, the brain must therefore 
rely on an internal estimate of the auditory feedback and use reafferent feedback only to correct this internal estimate. When an utterance is produced, an efference copy of the motor command is used to make a prediction of the current articulatory state (e.g. state of the vocal tract) and the subsequent sensory outcome. As long as there is no mismatch between the predicted and the reafferent feedback, the brain can rely on its internal estimate. However, when there is a mismatch, the brain generates an error signal to correct its internal estimate and the necessary motor commands to produce the intended utterance. In our paradigm, an artificially introduced delay generates a continuous mismatch between the predicted and the reafferent feedback. Specifically, the reafferent feedback matches the previous articulatory state rather than the current articulatory state. In this case, the issuing of new motor commands must be delayed, which would explain the slowing down of speech production. We found that producing single words with DAF elicited a nominal slowing down effect and increased neural responses only in auditory but not in motor regions. However, when subjects produced sentences with DAF, this longer and more complex stimulus elicited a much prominent slowing down effect and increased neural responses in auditory as well as motor regions.

Neural responses to DAF were enhanced in STG, SMG, dPreCG and IFG, which are anatomically connected by one of the major language pathways known as the superior longitudinal fasciculus $[36,37]$. These regions are typically modeled as the main components of the dorsal stream for speech that is responsible for sensorimotor integration and auditory feedback processing [38]. In support of these theoretical models, clinical reports demonstrated that posterior STG and SMG damage are implicated in conduction aphasia [39] and patients with conduction aphasia are less affected by DAF [40] indicating the involvement of this regions in feedback processing. 
Our analysis of the time course of responses revealed that response enhancement to DAF started in STG and closely followed by dPreCG providing further evidence for a functional correspondence between the two regions. $\mathrm{dPreCG}$ is a complex functional region implicated in auditory, motor and visual speech processing [41-44]. It is known to be activated not only during speaking but also during passive listening suggesting a role in mapping acoustic speech features onto the corresponding articulatory movements. We predict that $\mathrm{dPreCG}$ may be the hub for generating the internal estimate of the speech output by processing both the efference copy and auditory error signals during speech production.

Interestingly, response enhancement in dPreCG during auditory feedback processing has never been reported previously. Studies that altered auditory feedback using pitch perturbation demonstrated enhanced responses in ventral parts of the precentral gyrus, which correlated with the compensatory vocal adjustments [12-14]. In our experiments, vPreCG did not show any response enhancement neither for word nor for sentence production with DAF. It could be the case that vocal control that requires pitch adjustments activate vPreCG, while vocal control that requires long-term maintenance of other prosodic features such as tempo, rhythm and pause, activate dPreCG. dPreCG is well known to be involved in movement planning and execution $[45,46]$. Previous studies reported increased activation in this region when subjects produced complex syllable sequences, suggesting a role in the planning and production of long speech utterances with appropriate syllable timing [47]. Considering that dPreCG activation occurred only when subjects produced sentences with DAF, we predict that it plays a critical role in maintaining prosody and speech fluency during the articulation of long utterances.

Our results showed that the maximal disruption of speech occurred at $200 \mathrm{~ms}$ feedback delay, which agrees with previous behavioral reports [18-20, 48, 49]. The dynamics of the 
cortical speech network can provide an explanation for this effect. Previous ECoG studies showed that IFG is activated before articulation onset and remained silent during articulation, while motor cortex is activated both before and during articulation. These studies suggested that IFG produces an articulatory code that is subsequently implemented by the motor cortex and reported a $200 \mathrm{~ms}$ temporal lag between IFG and motor cortex activation [50, 51]. A feedback delay in the same order of this temporal lag likely interrupts propagation of the articulatory code from IFG to motor cortex, thereby disrupting speech. Unlike prior reports, we found sustained IFG activity throughout speech production, however this was during DAF where sustained IFG recruitment may be necessary to support compensatory speech correction. The onset of IFG activation was seen in conjunction with dPreCG, however sensitivity to DAF was seen at two distinct time periods with an early recruitment of dPreCG and much later involvement of IFG.

Behavioral paradigms that manipulate auditory feedback have been widely used for decades to understand speech motor control, however the cortical dynamics underlying this process remained largely unknown. We elucidate the magnitude, timing and spatial distribution of the neural responses that encode the mismatch between produced speech and its perceived feedback. Our results highlighted STG, SMG, IFG and dPreCG as critical players in detection and correction of vocalization errors. Specifically, we find that $\mathrm{dPreCG}$ is a selective region which is recruited immediately when auditory feedback becomes unreliable and production more effortful, implicating it in auditory-motor mapping that underlies vocal monitoring of human speech.

\section{Materials and methods}

\section{Subject information}


All experimental procedures were approved by the NYU Grossman School of Medicine Institutional Review Board. The review board considered: 1) the risks and anticipated benefits (if any) to subjects 2) the selection of subjects 3) the procedures for securing and documenting informed consent 4) the safety of subjects 5) the privacy of subjects and confidentiality of the data. The approval is for study i14-02101 and NYU Grossman School of Medicine Federal wide Assurance number is FWA00004952. All patients provided written consent prior to the study as well as an additional oral consent at the start of the experiment.

15 neurosurgical epilepsy patients ( 8 females, mean age: 34, 2 right, 9 left and 4 bilateral hemisphere coverage) implanted with subdural and depth electrodes provided informed consent to participate in the research protocol. Electrode implantation and location were guided solely by clinical requirements. 3 patients were consented separately for higher density clinical grid implantation, which provided denser sampling of underlying cortex.

\section{Experiment setup}

Subjects were tested while resting in their hospital bed in the epilepsy-monitoring unit. Visual stimuli were presented on a laptop screen positioned at a comfortable distance from the subject. Auditory stimuli were presented through earphones (Bed Phones On-Ear Sleep Headphones Generation 3) and subjects' voice was recorded using an external microphone (Zoom H1 Handy Recorder).

\section{Delayed auditory feedback experiment}

The experiment consisted of a word-reading session and a sentence-reading session. 10 different 3-syllable words (e.g. document) were used in the word-reading session, and 6 different 8-word sentences (e.g. The cereal was fortified with vitamins and nutrients) were used in the sentence-reading session. Text stimuli were visually presented on the screen and subjects were 
instructed to read them out loud. As subjects spoke, their voices were recorded using the laptop's internal microphone, delayed at 4 different amounts (no delay, 50, 100, 200ms) using custom script (MATLAB, Psychtoolbox-3) and played back to them through earphones. A TTL pulse marking the onset of a stimulus, the delayed feedback voice signal (what the patient heard) and the actual microphone signal (what the patient spoke) were fed in to the EEG amplifier as an auxiliary input in order to acquire them in sync with the EEG samples. Trials, which consisted of different stimulus-delay combinations, were presented randomly ( 3 to 8 repetitions) with a 1 second inter-trial-interval.

\section{Electrocorticography (ECoG) recording}

ECoG was recorded from implanted subdural platinum-iridium electrodes embedded in flexible silicon sheets $(2.3 \mathrm{~mm}$ diameter exposed surface, $8 \times 8$ grid arrays and 4 to 12 contact linear strips, 10 mm center-to-center spacing, Ad-Tech Medical Instrument, Racine, WI) and penetrating depth electrodes $(1.1 \mathrm{~mm}$ diameter, $5-10 \mathrm{~mm}$ center-to-center spacing 1 x 8 or $1 \times 12$ contacts, Ad-Tech Medical Instrument, Racine, WI). Three subjects consented to a research hybrid grid implanted which included 64 additional electrodes between the standard clinical contacts $(16 \times 8$ grid with sixty-four $2 \mathrm{~mm}$ macro contacts at $8 \times 8$ orientation and sixty-four 1 $\mathrm{mm}$ micro contacts in between, providing $10 \mathrm{~mm}$ center-to-center spacing between macro contacts and $5 \mathrm{~mm}$ center-to-center spacing between micro/macro contacts, PMT corporation, Chanassen, MN). Recordings were made using one of two amplifier types: NicoletOne amplifier (Natus Neurologics, Middleton, WI), bandpass filtered from 0.16-250 Hz and digitized at 512 Hz. Neuroworks Quantum Amplifier (Natus Biomedical, Appleton, WI) recorded at 2048 $\mathrm{Hz}$, bandpass filtered at $0.01682 .67 \mathrm{~Hz}$ and then downsampled to $512 \mathrm{~Hz}$. A two-contact subdural strip facing toward the skull near the craniotomy site was used as a reference for 
recording and a similar two-contact strip screwed to the skull was used for the instrument ground. Electrocorticography and experimental signals (trigger pulses that mark the appearance of visual stimuli on the screen, microphone signal from speech recordings and auditory playback signal that was heard by the patients through earphones) were acquired simultaneously by the EEG amplifier in order to provide a fully synchronized dataset. Recorded microphone and feedback signals were analyzed to ensure that the temporal delay manipulation by our MATLAB code produced the intended delay.

\section{Electrode localization}

Electrode localization in subject space as well as MNI space was based on co-registering a preoperative (no electrodes) and postoperative (with electrodes) structural MRI (in some cases a postoperative $\mathrm{CT}$ was employed depending on clinical requirements) using a rigid-body transformation. Electrodes were then projected to the surface of cortex (preoperative segmented surface) to correct for edema induced shifts following previous procedures [27] (registration to MNI space was based on a non-linear DARTEL algorithm [28]). Within subject anatomical locations of electrodes was based on the automated FreeSurfer segmentation of the subject's preoperative MRI. All middle and caudal superior temporal gyrus electrodes were grouped as superior temporal gyrus (STG), all parsopercularis and pars triangularis electrodes were grouped as inferior frontal gyrus (IFG) electrodes. Precentral electrodes with a z coordinate smaller than \pm 40 were grouped as ventral precentral gyrus (vPreCG), and those with a z coordinate larger than or equal to \pm 40 were grouped as dorsal precentral gyrus (dPreCG) together with electrodes located in caudal middle frontal gyrus.

\section{Neural data analysis}

A common average reference was calculated by subtracting the average signal across all 
electrodes from each individual electrode's signal (after rejection of electrodes with artifacts caused by line noise, poor contact with cortex and high amplitude shifts). Continuous data streams from each channel were epoched into trials (from $-1.5 \mathrm{~s}$ to $3.5 \mathrm{~s}$ for word stimuli and from $-1.5 \mathrm{~s}$ to $5.5 \mathrm{~s}$ for sentence stimuli with respect to speech onset). Line noise at 60,120 and $180 \mathrm{~Hz}$ were filtered out and the data was transformed to time-frequency space using the multitaper method (MATLAB, FieldTrip toolbox) with 3 Slepian tapers; frequency windows from 10 to $200 \mathrm{~Hz}$; frequency steps of $5 \mathrm{~Hz}$; time steps of $10 \mathrm{~ms}$; temporal smoothing of $200 \mathrm{~ms}$; frequency smoothing of $\pm 10 \mathrm{~Hz}$. The high gamma broadband response $(70-150 \mathrm{~Hz})$ at each time point following stimulus onset was measured as the percent signal change from baseline, with the baseline calculated over all trials in a time window from -500 to $-100 \mathrm{~ms}$ before stimulus onset. High gamma response duration for each electrode was measured by calculating the time difference at full width quarter maximum of the response curve.

\section{Electrode selection}

We recorded from a total of 1693 subdural and 608 depth electrode contacts in 15 subjects. Electrodes were examined for speech related activity defined as significant high gamma broadband responses. For auditory repetition and DAF word-reading tasks, electrodes that showed significant response increase ( $\mathrm{p}<10^{-4}$, unpaired t-test) either before $(-0.5$ to $0 \mathrm{~s})$ or after speech onset ( 0 to $0.5 \mathrm{~s}$ ) with respect to a baseline period ( -1 to $-0.6 \mathrm{~s}$ ) and at the same time had a large signal-to-noise ratio $(\mu / \sigma>0.7)$ during either of these time windows were selected. For DAF sentence-reading task, the same criteria were applied, except the time window after speech onset was longer ( 0 to $3 \mathrm{~s}$ ). Electrode selection was first performed for each task separately, then electrodes that were commonly selected for both tasks were further analyzed.

\section{Clustering analysis}


Non-negative matrix factorization (NMF) was used to identify major response patterns across different brain regions during speech production. NMF is an unsupervised dimensionality reduction (or clustering) technique that reveals the major patterns in the data without specifying any features [29]. We performed the clustering analysis using the data from the word-reading with DAF task, since this data set contained a large number of trials. We combined responses from all subjects by concatenating trials and electrodes forming a large data matrix A (electrodes-by-timepoints). Matrix A was factorized into two matrices $\mathrm{W}$ and $\mathrm{H}$ by minimizing the root mean square residual between $\mathrm{A}$ and $\mathrm{W}^{*} \mathrm{H}$ (nnmf function in MATLAB). Factorization was performed based on two clusters to represent the two major predicted speech related components in the brain; auditory and motor.

\section{Dynamic time warping analysis}

For each trial of the DAF sentence-reading task, the speech spectrogram was averaged across frequencies. Then, the mean spectrograms were averaged across trials of the same sentence stimuli (e.g. averaged over Sentence \#1 trials). Dynamic time warping (DTW) was performed separately for different sentence stimuli. The first 900 time points of the resulting DTW paths were applied to the neural response signal at each trial (representing approximately 9 seconds in the common warped time). Finally, the transformed neural responses were averaged across trials for each sentence stimuli. This procedure was performed to compare two conditions that resulted in the largest neural response difference (no delay versus $200 \mathrm{~ms}$ delay).

\section{Statistical analysis}

The effect of DAF on speech behavior was determined by performing one-way ANOVA across subjects using articulation duration of words and sentences as the dependent variable and delay condition as the independent variable. To determine a significant difference in the 
amplitude of neural response between conditions, the average high gamma activity in a specified time window was compared by performing one-way ANOVA across all trials in all electrodes using delay condition as the independent variable. Similarly, a significant difference in the duration of neural response was determined by performing one-way ANOVA across subjects using response duration as the dependent variable and delay condition as the independent variable. Significance levels were computed at a p-value of 0.01 . To compare sensitivity to DAF for word and sentence-reading, sensitivity indices were compared across electrodes using an unpaired t-test. To reveal how response enhancement to DAF changed across time during the sentence-reading task, we performed a one-way ANOVA at each time point using the neural response in each electrode as a dependent variable and delay as an independent variable. We performed a permutation test at each timepoint to assess a significance threshold for the F-value. We shuffled the delay condition labels 1000 times and performed an ANOVA for each timepoint at each iteration, then we set the threshold the 0.999 quantile of the F-value distribution.

\section{Acknowledgement}

We thank Zhuoran Huang and Qingyang Zhu for their assistance in analyzing voice recordings of the subjects.

\section{References}

1. Lombard E. Le signe de l'elevation de la voix. Ann Mal de L'Oreille et du Larynx. 1911:10119.

2. Zollinger SA, Brumm H. The Lombard effect. Curr Biol. 2011;21(16):R614-5.

3. Civier O, Tasko SM, Guenther FH. Overreliance on auditory feedback may lead to sound/syllable repetitions: simulations of stuttering and fluency-inducing conditions with a neural model of speech production. J Fluency Disord. 2010;35(3):246-79. 
4. Goldberg TE, Gold JM, Coppola R, Weinberger DR. Unnatural practices, unspeakable actions: a study of delayed auditory feedback in schizophrenia. Am J Psychiatry. 1997;154(6):858-60.

5. Hardy CJD, Bond RL, Jaisin K, Marshall CR, Russell LL, Dick K, et al. Sensitivity of Speech Output to Delayed Auditory Feedback in Primary Progressive Aphasias. Front Neurol. 2018;9:894.

6. Lin IF, Mochida T, Asada K, Ayaya S, Kumagaya S, Kato M. Atypical delayed auditory feedback effect and Lombard effect on speech production in high-functioning adults with autism spectrum disorder. Front Hum Neurosci. 2015;9:510.

7. Liu H, Wang EQ, Metman LV, Larson CR. Vocal responses to perturbations in voice auditory feedback in individuals with Parkinson's disease. PLoS One. 2012;7(3):e33629.

8. Hickok G, Houde J, Rong F. Sensorimotor integration in speech processing: computational basis and neural organization. Neuron. 2011;69(3):407-22.

9. Houde JF, Nagarajan SS. Speech production as state feedback control. Frontiers in human neuroscience. 2011;5:82.

10. Tourville JA, Guenther FH. The DIVA model: A neural theory of speech acquisition and production. Lang Cogn Process. 2011;26(7):952-81.

11. Eliades SJ, Wang X. Neural substrates of vocalization feedback monitoring in primate auditory cortex. Nature. 2008;453(7198):1102-6.

12. Houde JF, Jordan MI. Sensorimotor adaptation in speech production. Science. 1998;279(5354):1213-6.

13. Jones JA, Munhall KG. Perceptual calibration of F0 production: evidence from feedback perturbation. J Acoust Soc Am. 2000;108(3 Pt 1):1246-51.

14. Niziolek CA, Guenther FH. Vowel category boundaries enhance cortical and behavioral responses to speech feedback alterations. J Neurosci. 2013;33(29):12090-8.

15. Behroozmand R, Karvelis L, Liu H, Larson CR. Vocalization-induced enhancement of the auditory cortex responsiveness during voice F0 feedback perturbation. Clin Neurophysiol. 2009;120(7):1303-12.

16. Behroozmand R, Shebek R, Hansen DR, Oya H, Robin DA, Howard MA, 3rd, et al. Sensorymotor networks involved in speech production and motor control: an fMRI study. Neuroimage. 2015;109:418-28.

17. Tourville JA, Reilly KJ, Guenther FH. Neural mechanisms underlying auditory feedback control of speech. Neuroimage. 2008;39(3):1429-43.

18. Black JW. The effect of delayed side-tone upon vocal rate and intensity. Journal of Speech and Hearing Disorders. 1951;16(1):56-60.

19. Fairbanks G. Selective vocal effects of delayed auditory feedback. Journal of speech and hearing disorders. 1955;20(4):333-46.

20. Lee BS. Effects of delayed speech feedback. The Journal of the Acoustical Society of America. 1950;22(6):824-6.

21. Blanchet P, Hoffman P. Factors influencing the effects of delayed auditory feedback on dysarthric speech associated with Parkinson's disease. Commun Disord Deaf Stud Hearing Aids. 2014;2(106):2.

22. Bloodstein O. A handbook on stuttering. 1969.

23. Kalinowski J, Stuart A, Sark S, Armson J. Stuttering amelioration at various auditory feedback delays and speech rates. International Journal of Language \& Communication Disorders. 1996;31(3):259-69. 
24. Hashimoto Y, Sakai KL. Brain activations during conscious self-monitoring of speech production with delayed auditory feedback: an fMRI study. Hum Brain Mapp. 2003;20(1):22-8. 25. Hirano S, Kojima H, Naito Y, Honjo I, Kamoto Y, Okazawa H, et al. Cortical processing mechanism for vocalization with auditory verbal feedback. Neuroreport. 1997;8(9-10):2379-82. 26. Takaso H, Eisner F, Wise RJ, Scott SK. The effect of delayed auditory feedback on activity in the temporal lobe while speaking: a positron emission tomography study. J Speech Lang Hear Res. 2010;53(2):226-36.

27. Yang AI, Wang X, Doyle WK, Halgren E, Carlson C, Belcher TL, et al. Localization of dense intracranial electrode arrays using magnetic resonance imaging. Neuroimage.

2012;63(1):157-65.

28. Ashburner J. A fast diffeomorphic image registration algorithm. Neuroimage. 2007;38(1):95113.

29. Ding C, He X, Simon HD, editors. On the equivalence of nonnegative matrix factorization and spectral clustering. Proceedings of the 2005 SIAM international conference on data mining; 2005: SIAM.

30. Mukamel R, Gelbard H, Arieli A, Hasson U, Fried I, Malach R. Coupling between neuronal firing, field potentials, and FMRI in human auditory cortex. Science. 2005;309(5736):951-4. 31. Nir Y, Fisch L, Mukamel R, Gelbard-Sagiv H, Arieli A, Fried I, et al. Coupling between neuronal firing rate, gamma LFP, and BOLD fMRI is related to interneuronal correlations. Current biology. 2007;17(15):1275-85.

32. Ray S, Maunsell JH. Different origins of gamma rhythm and high-gamma activity in macaque visual cortex. PLoS Biol. 2011;9(4):e1000610.

33. Hamilton LS, Edwards E, Chang EF. A spatial map of onset and sustained responses to speech in the human superior temporal gyrus. Current Biology. 2018;28(12):1860-71. e4. 34. Swink S, Stuart A. The effect of gender on the N1-P2 auditory complex while listening and speaking with altered auditory feedback. Brain and language. 2012;122(1):25-33.

35. Toyomura A, Miyashiro D, Kuriki S, Sowman PF. Speech-Induced Suppression for Delayed Auditory Feedback in Adults Who Do and Do Not Stutter. Frontiers in Human Neuroscience. 2020;14:150.

36. Chang EF, Raygor KP, Berger MS. Contemporary model of language organization: an overview for neurosurgeons. J Neurosurg. 2015;122(2):250-61.

37. Makris N, Kennedy DN, McInerney S, Sorensen AG, Wang R, Caviness VS, Jr, et al. Segmentation of Subcomponents within the Superior Longitudinal Fascicle in Humans: A Quantitative, In Vivo, DT-MRI Study. Cerebral Cortex. 2004;15(6):854-69.

38. Hickok G, Poeppel D. The cortical organization of speech processing. Nature reviews neuroscience. 2007;8(5):393-402.

39. Fridriksson J, Kjartansson O, Morgan PS, Hjaltason H, Magnusdottir S, Bonilha L, et al. Impaired speech repetition and left parietal lobe damage. J Neurosci. 2010;30(33):11057-61. 40. Boller F, Marcie P. Possible role of abnormal auditory feedback in conduction aphasia. Neuropsychologia. 1978;16(4):521-4.

41. Callan DE, Jones JA, Callan A. Multisensory and modality specific processing of visual speech in different regions of the premotor cortex. Front Psychol. 2014;5:389.

42. Cheung C, Hamilton LS, Johnson K, Chang EF. Correction: The auditory representation of speech sounds in human motor cortex. Elife. 2016;5.

43. Ozker M, Yoshor D, Beauchamp MS. Frontal cortex selects representations of the talker's mouth to aid in speech perception. Elife. 2018;7. 
44. Pulvermuller F, Huss M, Kherif F, Moscoso del Prado Martin F, Hauk O, Shtyrov Y. Motor cortex maps articulatory features of speech sounds. Proc Natl Acad Sci U S A.

2006;103(20):7865-70.

45. Grafton S, Fagg A, Arbib M. Dorsal premotor cortex and conditional movement selection: A PET functional mapping study. Journal of Neurophysiology. 1998.

46. Thoenissen D, Zilles K, Toni I. Movement preparation and motor intention: an event-related fMRI study. Journal of Neuroscience. 2002;22:9248-60.

47. Bohland JW, Guenther FH. An fMRI investigation of syllable sequence production.

Neuroimage. 2006;32(2):821-41.

48. Stuart A, Kalinowski J, Rastatter MP, Lynch K. Effect of delayed auditory feedback on normal speakers at two speech rates. J Acoust Soc Am. 2002;111(5 Pt 1):2237-41.

49. Yamamoto K, Kawabata H. Adaptation to delayed auditory feedback induces the temporal recalibration effect in both speech perception and production. Exp Brain Res.

2014;232(12):3707-18.

50. Flinker A, Korzeniewska A, Shestyuk AY, Franaszczuk PJ, Dronkers NF, Knight RT, et al. Redefining the role of Broca's area in speech. Proc Natl Acad Sci U S A. 2015;112(9):2871-5. 51. Magrassi L, Aromataris G, Cabrini A, Annovazzi-Lodi V, Moro A. Sound representation in higher language areas during language generation. Proc Natl Acad Sci U S A. 2015;112(6):186873. 